\title{
Le conflit frontalier Cameroun-Nigeria dans le lac Tchad : les enjeux de l'île de Darak, disputée et partagée
}

The border conflict between Cameroon and Nigeria in the Lake Chad: the stakes around the Darak Island, disputed and shared

Darak, conflicto fronterizo, fronteras, Camerún, Nigeria, finales del s. XX

\section{Halirou ABDOURAMAN}

\section{(2) OpenEdition}

Journals

Édition électronique

URL : http://journals.openedition.org/conflits/17311

DOI : $10.4000 /$ conflits. 17311

ISSN : $1777-5345$

Éditeur :

CCLS - Centre d'études sur les conflits lilberté et sécurité, L'Harmattan

Édition imprimée

Date de publication : 20 décembre 2008

Pagination : $57-76$

ISBN : 1157-966X

ISSN : 1157-996X

Référence électronique

Halirou ABDOURAMAN, «Le conflit frontalier Cameroun-Nigeria dans le lac Tchad : les enjeux de lîle de Darak, disputée et partagée », Cultures \& Conflits [En ligne], 72 I hiver 2008, mis en ligne le 19 mai 2009, consulté le 30 mars 2021. URL : http://journals.openedition.org/conflits/17311 ; DOI : https:// doi.org/10.4000/conflits. 17311 


\title{
Le conflit frontalier Cameroun-Nigeria dans le lac Tchad: les enjeux de l'île de Darak, disputée et partagée
}

\section{ABDOURAMAN Halirou}

Abdouraman Halirou est enseignant-chercheur au département d'histoire de l'université de Ngaoundéré. Il travaille notamment sur la problématique des frontières (politiques, géopolitiques, géostratégiques, socioculturelles internes et internationales), avec un accent sur l'Afrique subsabarienne. Il a notamment publié "Yaya Daïrou: Lamido de Marona (1943-1958)» in Thierno Monctar Bab (dir.), Acteurs de l'histoire au Nord-Cameroun, Ngaoundéré-Anthropos, vol. 1, 1999.

\begin{abstract}
A la fin des années 1970 et au début des années 1980, une sécheresse permanente ravage tout le Sahel africain. Outre de nombreuses morts, on constate que des milliers de personnes migrent d'une région à une autre, en dépit des frontières interétatiques existantes. C'est dans ce contexte de crise que des populations nigérianes se mettent à occuper certaines îles camerounaises surgies à la suite de l'assèchement du lac Tchad. Peu après, l'administration nigériane du Borno State appuie cette occupation en y établissant sa domination sur des territoires qui, théoriquement, se trouvent en terre camerounaise. Chose curieuse, l'installation nigériane ne semble pas être considérée comme étrangère. En effet, au moment ou les deux Etats se disputent la paternité de certaines îles du lac Tchad, des populations d'origines diverses (camerounaises, nigérianes, maliennes, tchadiennes, centrafricaines, etc.), liées par l'exploitation des ressources du lac, notamment le poisson, y vivent en paix.
\end{abstract}

Dès lors, le propos de cette réflexion est de tenter de percer les enjeux de ce paradoxe : comment comprendre qu'au moment où le Cameroun et le Nigeria se disputent la paternité et le contrôle de Darak (en réalité, du lac Tchad), les populations frontalières et surtout camerounaises vivent en paix, partageant son foncier utile et cautionnent d'une manière ou d'une autre la domination nigériane ? Peut-on vraiment parler de frontières fixes dans cette région doublement instable ? 
Il s'agit ainsi de comprendre les enjeux du conflit de frontière né aux abords du lac entre le Cameroun et le Nigeria au début des années 1990. Par ailleurs, ceci nous permettra de saisir les modes de régulations transfrontalières ayant permis aux populations de développer une intégration pacifique par le bas, l'objectif fonctionnel de cette étude étant de suggérer le dépassement des frontières étanches. Ce but, pour être atteint, appelle nécessairement un examen de la dynamique de ce conflit frontalier depuis ses débuts jusqu'à sa résolution. Dès lors, une analyse comparative des politiques des frontières du Cameroun et du Nigeria permettra de dégager leurs impacts respectifs sur la gestion des zones frontalières. Enfin, une présentation de la continuité du système de coopération économique et sociale entre les populations de Darak pendant et après l'occupation nigériane mettra à nu le caractère véritablement international, voire sans frontière, de cet espace.

\section{Le conflit frontalier Cameroun-Nigeria : causes, résolution et impact sur l'accès au lac Tchad et le contrôle de ses ressources}

Depuis l'époque précoloniale, le lac Tchad appelé Koura ("grand») par les Kanouri, constitue un enjeu géopolitique majeur. Il constitue un véritable rempart à l'avancée du désert saharien, malgré la diminution progressive de sa surface. Dans ce contexte, le lac Tchad fait l'objet d'âpres disputes qui remonteraient aux XIIIe-XIVe siècles.

Cette période est marquée par la «migration territoriale 1 » du Kanem vers les berges occidentales du lac, à la suite de l'invasion boulala confortée par une guerre civile entre les Idrissides et les Daoudiens ${ }^{2}$. Marquant les débuts institutionnels de l'empire du Bornou, cet événement ouvre ainsi la voie aux conflits et aux luttes pour l'accès et le contrôle des ressources du lac Tchad et des circuits commerciaux qui s'y rattachent. D'une manière ou d'une autre, les conquêtes du May Idris Aloma au XVIe siècle, qui ont poussé le plus loin possible les frontières du Bornou (obligeant par exemple les Tliksé du Wandala plus au Sud à changer de capitale), entre dans cette logique. Dès lors, la soumission des principautés Kotoko vassalisées offre au Bornou l'Ouest et le Sud du lac.

1. Abdouraman H., "Frontières et découpages territoriaux dans l'extrême-Nord du Cameroun : enjeux et implications $\left(\mathrm{XIV}^{\mathrm{e}}-\mathrm{XX}^{\mathrm{e}}\right.$ siècle) », thèse de doctorat / $\mathrm{PhD}$ d'Histoire, université de Ngaoundéré, 2007.

2 . Zeltner J.-C., Pages d'histoire du Kanem, Paris, L'Harmattan, 1980. 
Carte de localisation de Darak dans le lac Tchad ${ }^{3}$

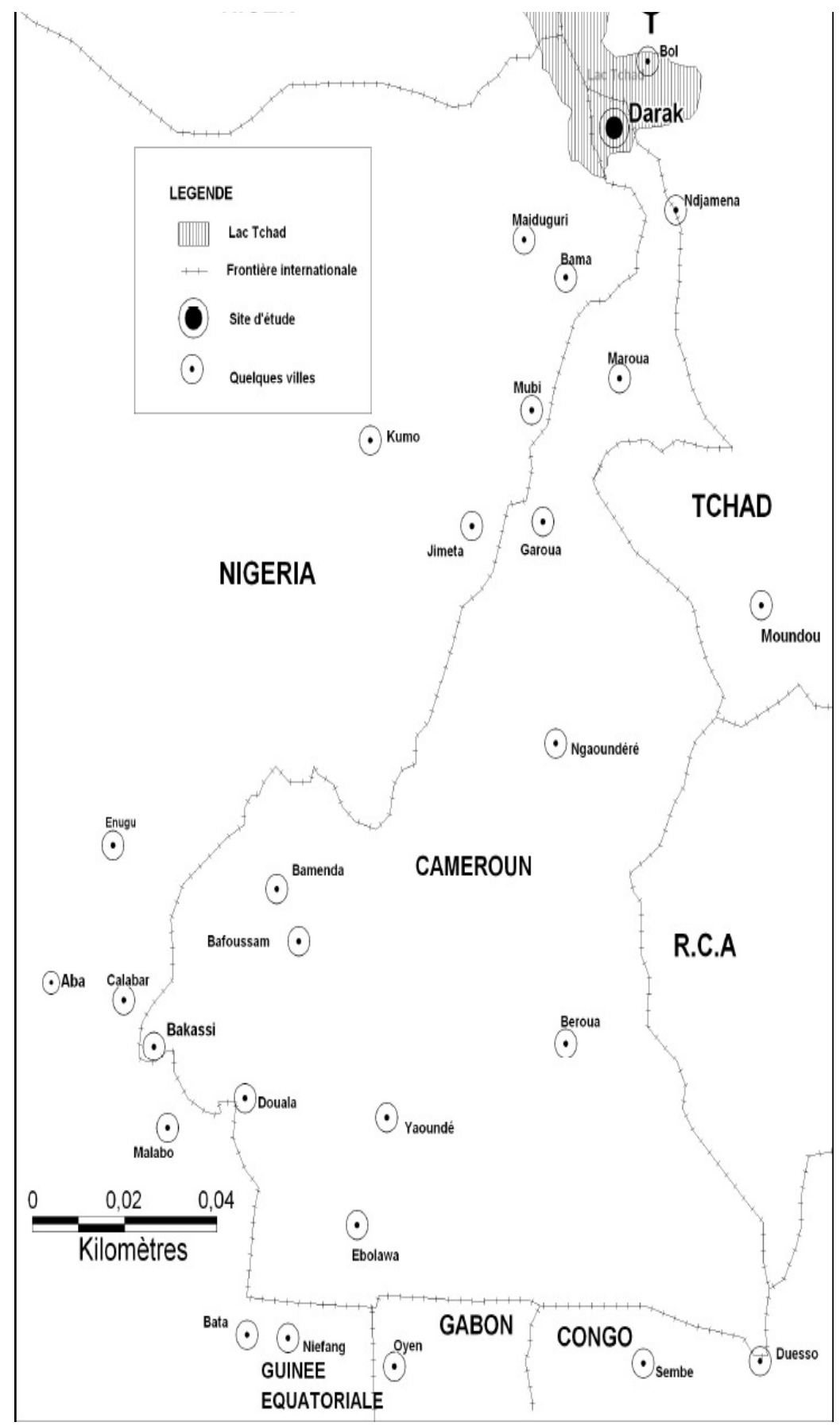

3. Source : Encyclopédie virtuelle Encarta, 2007. Réalisation: Baska Toussia Daniel, attaché d'enseignement et de recherche (ATER) au Département de géographie, université de Ngaoundéré, 4 novembre 2008. 
Cependant, le contexte de guerres permanentes, associé à l'assèchement progressif de cette étendue d'eau, ne permettait point de tracer des frontières étanches dans la zone. D’ailleurs, comme l'ont bien souligné de nombreux spécialistes de la limologie ${ }^{4}$, l'Afrique précoloniale ne connaît guère de frontières linéaires, en dehors des obstacles naturels (grands fleuves comme le Logone, séparant le Bornou du Baguirmi, chaines montagneuses) et des ouvrages défensifs, à l'instar des fortifications ceinturant la plupart des cités, et notamment les principautés Kotoko. Ce sont des frontières dites «zonales » ou « zones frontières », très mouvantes au gré des conquêtes. Malgré les nombreux dangers qui s'y trouvent (brigands, fauves, lieux de batailles, etc.), les fondateurs des grands Etats conquérants n'ont pas développé de frontières étanches ${ }^{5}$.

De toutes les façons, les mouvements constants des hommes dans le bassin tchadien s'accomodaient fort peu de barrières frontalières telles qu'elles sont visibles de nos jours à certains endroits. Toutefois, les entités centralisées disposaient de mécanismes permettant de canaliser les déplacements humains. Ces dispositions organisationnelles, du fait de la pression des facteurs naturels (crises climatiques, mauvaises récoltes) et des enjeux politiques (nécessité de conquêtes, esclavage, accès / contrôle des ressources naturelles, mode de vie nomade de certains peuples, profondeur des liens socioculturels) ont fait du bassin tchadien en général et du lac Tchad en particulier, une zone ouverte 6 .

C'est dans ce milieu plus ou moins sans frontières que les colonisateurs européens vont tracer des limites fixes afin de délimiter clairement leurs zones d'influence réciproques. La conférence de Berlin de 1884-1885 en définit les fondements. Les accords bilatéraux, signés d'une part entre les Allemands et les Anglais et, d'autre part entre les Anglais et les Français, vont nettement définir ce qui deviendra plus tard la frontière séparant l'Etat nigérian de celui du Cameroun. Sur la base des latitudes et des longitudes, les coordonnées de cette «diade 7 » sont à la fois terrestres et lacustres. Dès lors, des missions d'abornement ont été initiées pour concrétiser les délimitations négociées, sans associer les populations locales concernées. Cependant, cette bonne volonté politique de démarcation frontalière ne sera pas effective dans le lac. Aussi, le retrait de ses eaux sera-t-il à la base de nombreux conflits.

4. Du radical «limes », la limologie est un concept développé par les géographes Paul Guichonnet et Claude Raffestin qui désigne l'ensemble des sciences qui analysent les frontières et les faits frontaliers. Voir Guichonnet P., Raffestin C., Géographie des frontières, Vendôme, PUF, 1974.

5 . Nous pensons en particulier à Idris Aloma du Kanem-Bornou et Ousman dan Fodio de l'empire peul de Sokoto.

6. En réalité, il s'agit d'un enchevêtrement de frontières mentales qui sont instables de par le caractère provisoire des liens de vassalité. La multiplication de ce type de frontières précaires et non inscrites sur le sol fait du bassin tchadien une zone sans frontière. Dès lors, le lac Tchad appartient momentanément au «plus fort ».

7. Foucher M., Fronts et frontières : un tour du monde géopolitique, Paris, Fayard, 2005. 
Le Cameroun et le Nigeria héritent donc de cette situation dualiste portant déjà des germes conflictuels. Comme le reste des pays africains, les deux pays voisins confirment le caractère intangible des frontières héritées de la colonisation ${ }^{8}$. A travers la Commission du bassin du lac Tchad (CBLT) instituée en 1962, des barres de fer distantes les unes des autres de $10 \mathrm{~km}$ sont alors implantées dans le lac pour servir d'éléments de démarcation effective de leur frontière. Or, le retrait progressif des eaux du lac Tchad change la donne.

En effet, libérant des terres exondées très fertiles, il donne lieu à de rudes compétitions pour l'accès et le contrôle de ce foncier utile. C'est dans ce contexte que les populations nigérianes, plus nombreuses et très entreprenantes, occupent les terres ainsi libérées et les îles qui se sont créées à l'issue des vidanges successives du lac. Située à l'extrémité septentrionale du Cameroun, et d'accès difficile en toute saison, particulièrement en saison des pluies, cette région n'est pas aisément administrée par l'Etat camerounais. Cela facilite l'«intégration » de la plus importante des îles du lac Tchad, Darak, dans le Borno State. Ainsi, pendant plusieurs années le drapeau vert-blanc-vert nigérian va flotter en terre camerounaise malgré les protestations de Yaoundé.

En réalité, ce conflit frontalier relève des enjeux géo-économique (contrôle du flux commercial et de ses retombées) et géopolitique (contrôle des migrations et de l'intégrité territoriale) liés au retrait des eaux du lac Tchad et à la migration de ses ressources (eaux, poissons, pâturages, foncier agricole) vers le Cameroun. En effet, la naissance même de Darak est liée à l'évolution régressive du lac Tchad. Comme le signale fort bien Saibou Issa :

«[...] dans ce processus historique, l'eau a joué et continue toujours de jouer un rôle fondamental dans l'attraction et la diffusion des peuples. [...] Dans leur quête des colonies, l'accès au lac Tchad fut l'un des objectifs majeurs des colonisateurs français, anglais et allemand ${ }^{9}$ ».

Dans le cas d'espèce, c'est toujours l'eau qui constitue l'enjeu majeur. Face à la permanence de la sécheresse depuis les années 1970, le lac Tchad libère progressivement d'importantes terres qui offrent de nombreuses richesses et potentialités agro-pastorales. De toute évidence, le lac Tchad actuel n'est que

8. Voir la Résolution $n^{\circ} 16$ de l'OUA, Caire 1964, relative à l'intangibilité des frontières africaines héritées de la colonisation. Ce principe sera à la base de toutes les résolutions des conflits frontaliers prononcées par la Cour internationale de Justice (CIJ) de la Haye : www.icj-cij.org

9. Notre traduction: "In this historical process, water has played and still continues to play a fundamemtal role in the attraction and diffusion of peoples [...]. In their quest for colonies, access to Lake Chad was one of the major objectives of the French, English and German colonizers", in Saibou I., "Access to Lake Chad and Nigeria-Cameroon Border Conflict: A Historical Perspective", in Castelein S., Otte A. (eds), Conflict and Co-operation Related to International Water Resources: Historical Perspectives, Selected Papers of the IWHA Conference on the role of water in history and development, Bergen, Norway, 10-12 août 2001, International Hydrological Programme, Technical Documents in Hydrology, n⿳62, 2002. 
le reste d'un vaste ensemble lacustre (le paléo-Tchad) dont la dernière extension remonte à l'Holocène. Marqué depuis par une évolution en dents de scie, il est, depuis le début du XXe siècle, en constante régression : $23500 \mathrm{~km}^{2}$ en 1962, $19000 \mathrm{~km}^{2}$ en $1972,9000 \mathrm{~km}^{2}$ en 1973. Ainsi, au milieu des années 1990, plus de 30 villages ont été créés par plus de 7000 Nigérians dans la zone camerounaise du lac Tchad. Dès lors, Darak, située à $35 \mathrm{~km}$ à l'Est de la frontière du Nigeria, aurait été initialement occupée par des pêcheurs nigérians qui ont suivi le retrait des eaux du lac; cela se serait déroulé à la fin des années 198010.

De ce fait, comme signalé plus haut, le bassin du lac Tchad est un espace ouvert, voire sans frontières ${ }^{11}$. C'est cette caractéristique doublée d'une apparence d'abandon presque général de ses zones frontalières par le Cameroun qui pousse les populations nigérianes, au demeurant plus nombreuses, à parcourir les rives $\mathrm{du}$ lac à la recherche des endroits les plus poissonneux. L'assèchement du lac fait que les ressources rares traversent les frontières séparant les Etats. Il est également à noter que c'est la partie nigériane du lac Tchad qui a le plus souffert des retraits des eaux. Par conséquent, les Nigérians ont été privés d'importantes richesses à la suite des sécheresses de 1972-1973 et de 1983-1985. La plus grande partie des activités liées à la pêche se faisait du côté nigérian. Au départ saisonniers, leurs séjours dans les régions poissonneuses du Cameroun finissent par s'éterniser. Alors, ils s'y implantent. D'île en île, ils investissent toute la partie camerounaise qui sera érigée en arrondissement de Darak.

Constituée de 21 villages et de 14 îles, Darak s'étend sur 658,60 km². Peuplée d'environ 20000 habitants en 2006, l'île a une densité de 75,90 habitants au km². Plusieurs groupes humains y cohabitent (Haoussa, Djoukoun, Margui, Mousgoum, Massa, Ibo, Arabe-Choa, Kotoko). Néanmoins, les Haoussa nigérians sont majoritaires. Comme le signale l'enquête réalisée par Tom Abdouraman, plus de $90 \%$ sont là pour pratiquer la pêche ${ }^{12}$. Principale activité de la région, la pêche draine d'importantes devises. Ainsi, depuis l'occupation nigériane de Darak, la production, qui était estimée à 100000 tonnes de poissons par an avant 1970, dépasse désormais 140000 tonnes. Le développement d'un commerce de poisson fait alors de Darak le cœur économique de la région. En effet, l'île de Darak en est la plaque tournante. Toutes les prises faites dans les autres îles sont rapportées et vendues à Darak. D’après les informations données en 2006 par le chef des pêcheurs, Al Hadji Hassan, plus de 700 sacs de $100 \mathrm{~kg}$ de poisson séché et 300 cartons de poisson fumé sont exportés vers Maiduguri ${ }^{13}$.

10. Abdouraman T., «Occupation de l'espace et gestion des ressources halieutiques dans la zone de Darak au lac Tchad », mémoire de maitrise de géographie, université de Ngaoundéré, 2006.

11. Abdouraman H., op. cit.

12. Abdouraman Tom, op. cit., p. 27.

13. Ibid. 
Cette importante activité est détenue par les négociants nigérians, la ville de Maiduguri en constituant le pôle. C'est ce qui justifie pleinement l'implantation de l'administration militaire nigériane à Darak. En effet, le 17 mai 1987, à la suite de la migration définitive de ses populations à Darak, le Nigeria décide de hisser son drapeau sur le territoire camerounais. Dès lors, une planification minutieuse de l'occupation d'une trentaine d'îles est alors organisée: Bachaka, Daba, Goré, Kendi, Kamouna, Katékimé, Kaforam, Dji-Bouniba, Hilé-Wanzam, Mourdas, Tororoya, Hilé-Adji, Goré-Tchandi, Goré al Goutoun, Lokonaira, Sokotram, Darak Gana, Faggé, Tchika’a, Ramin, Dorina, etc. ${ }^{14}$ Malgré les protestations camerounaises, la présence des forces militaires et administratives nigérianes dans la région de Darak perdure. Répondant aux plaintes du gouvernement camerounais formulées le 11 avril 1994 dans une note adressée à l'ambassadeur de la République fédérale du Nigeria à Yaoundé, Abuja soutient :

«Il est à la fois regrettable et inacceptable que Darak, qui a toujours fait partie du district de Wulgo dans le gouvernement local de Ngala de l'Etat du Bornou au Nigeria, et qui est depuis des temps immémoriaux administré en tant que tel, ne puisse être attribuée au territoire camerounais ${ }^{15}$.»

Cette réponse, qui viole les protocoles diplomatiques, témoigne de l'esprit impérialiste du géant africain. Et comme pour certifier la paternité nigériane, les chefs locaux des villages conquis, dont Darak, Bachaka, Katékimé, Kaforam, DjiBouniba, Hilé-Wanzam, Mourdas, Tororoya, Hilé-Adji, Goré-Tchandi, Goré al Goutoun, Lokonaira, Sokotram, sont enlevés et remplacés par des Nigérians, notamment Haoussa et Kanuri. En outre, des taxes exorbitantes sont infligées aux Camerounais : un cultivateur camerounais doit ainsi remettre l'équivalent de 10 sacs de sa production agricole aux forces nigérianes. A ces exactions sociales s'ajoute la substitution du drapeau camerounais par celui du Nigeria ${ }^{16}$.

Profitant de la plainte déposée le 29 mars 1994 à la CIJ pour règlement du différend frontalier au niveau de Bakassi, le Cameroun introduit une requête additionnelle le 6 juin 1994, sollicitant que l'organisme international précise définitivement le tracé de la frontière entre les deux pays, du lac Tchad à la mer, en invitant de ce fait le Nigeria à se retirer des terres illégalement occupées et à réparer les dommages matériels et moraux subis par les populations camerounaises.

14. Mondji L., « Le différend frontalier Cameroun-Nigeria dans la zone du lac Tchad avec accent sur Darak (dimension historique)», Rapport de recherche présenté dans le cadre de l'UV HS 304 «Sensibilisation à la recherche en Histoire ", université de Ngaoundéré, 2004, p. 10. Voir le site de la CIJ qui traite en détails de cette question : www.icj-cij.org

15. Notre traduction: "It is both unfortunate and unacceptable that Darak which has always been part and parcel of Wulgo District of Ngala Local Government area of Borno State of Nigeria and which has since immemorial time been administrated as such is no being claimed as part of Cameroon territory".

16. Mondji L., op. cit. 
Au terme du procès, la CIJ, avec l'arrêt du 10 octobre 2002, reconnaît l'héritage colonial en fixant de nouveau la frontière dans la région du lac Tchad, conformément à l'échange des notes franco-britanniques Henderson-Fleurion du 9 janvier 1931. Ainsi, les prétentions nigérianes sur Darak et les villages environnants sont rejetées. Le Nigeria est par conséquent invité à retirer, sans condition et dans les plus brefs délais, ses forces armées et sa police de la presqu'île ${ }^{17}$. En réalité, comme le note Saïbou Issa, «le verdict de la CIJ ne f[ai]t que confirmer la souveraineté du Cameroun sur la région de Darak et le tracé de la frontière lacustre tel que défini par les experts mandatés par la CBLT 18 ».

En effet, le 22 mai 1964, le traité instituant la Commission du bassin du lac Tchad, signé deux ans plus tôt à Fort-Lamy par les chefs d'Etat et de gouvernement du Cameroun, du Niger, du Nigeria et du Tchad, est approuvé. Pour ses pères fondateurs, la CBLT est chargée « d'une part d'organiser la gestion commune des eaux, des terres et des ressources naturelles $d u$ bassin $d u$ lac Tchad et d'autre part de coordonner le développement régional 19 ".

L'objectif inavoué est, à dire vrai, de garantir la paix en évitant les conflits pouvant surgir de l'exploitation des ressources de la région. D'ailleurs, les multiples bouleversements qu'a connus la zone à l'époque précoloniale peuvent réveiller des revendications territoriales, dans la mesure où la partition coloniale unilatérale ne satisfait pas tout le monde, et en particulier le Nigeria, héritier du Bornou, suzerain des terres à l'Ouest et au Sud du lac Tchad.

Fort de sa puissance financière, le Nigeria initie, contrairement aux principes multilatéraux de la CBLT, l'exploitation intensive des eaux du lac 20.

Le Cameroun rétorque en instituant la riziculture irriguée avec la Société d'exploitation et de modernisation de la riziculture de Yagoua (SEMRY). Ces activités économiques illégales, associées aux rudesses du climat contribuent d'une manière ou d'une autre à l'amenuisement des eaux du lac. Dès lors, les compétitions entre nationaux (et même des populations venues de l'Afrique de l'Ouest) pour l'accès aux ressources rares (eau, foncier pastoral et agricole, poisson) deviennent plus violentes. C'est ainsi qu'en 1983, soldats nigérians et tchadiens s'immiscent dans ces luttes et s'affrontent. Au-delà de la résolution du conflit tchado-nigérian, la CBLT décide alors de clarifier une fois pour toutes les frontières des Etats dans le lac Tchad.

17. Par ailleurs, la CIJ, s'attachant à la convention anglo-allemande du 11 mars 1913, reconnaît la souveraineté camerounaise sur Bakassi. Néanmoins, la paternité de certaines localités sur la frontière terrestre, est reconnue au Nigeria. Voir : www.icj-cij.org

18. Saïbou I., «Le mécanisme multilatéral de la CBLT pour la résolution des conflits frontaliers et la sécurité dans le bassin du lac Tchad ", Enjeux : bulletin d'analyses géopolitiques pour l'Afrique centrale, $\mathrm{n}^{\circ} 22$, janvier-mars 2005, pp. 31-33.

19. Ibid

20. Il s'agit de la mise en place du Chad Basin Irrigation Project (CBDA) au début des années 1970. CBLT 0045, PV de la 4e Conférence des chefs d'Etat, Lagos, 1983. 
En 1962 déjà, les présidents Ahmadou Ahidjo, Diori Hamani, Tafawa Balewa et François Tombalbaye ont reconnu le découpage colonial du lac ${ }^{21}$. La frontière camerouno-nigérianne est énoncée à l'origine par la convention germano-britannique de 1893. Elle est révisée et actualisée après la Première Guerre mondiale à travers le traité Milner-Simon du 10 juillet 1919. Dans cette déclaration francobritannique, qui s'est appuyée sur la carte Moisel allemande ${ }^{22}$, la frontière part du point de rencontre des trois anciennes frontières britannique, française et allemande située dans le lac à $13^{\circ} 5$ de latitude nord et approximativement $14^{\circ} 5$ de longitude est de Greenwich. La modification concerne essentiellement le point double (Cameroun-Nigeria) déplacé à l'Est du fait de l'établissement des mandats distincts au bénéfice de la France et de la Grande-Bretagne. L'accord ThompsonMarchand du 9 janvier 1930 ne change pas cette délimitation lacustre ${ }^{23}$.

C'est sur la base de ces délimitations coloniales que s'est appuyée l'équipe conjointe des experts de la CBLT devant suivre les travaux de démarcation de la frontière lacustre. Entamé en 1988, le bornage est réalisé à 98,5 \% en 1990 24. Il a été souvent compromis, compte tenu des manœuvres nigérianes pour la résolution bilatérale des différends frontaliers. De plus, l'abornement s'est compliqué du fait de l'occupation, dès le début de l'année 1988, de la zone de Darak par les troupes nigérianes qui ont profité de la suspension des travaux de démarcation, entre septembre 1988 et janvier 1989. Malgré les différends frontaliers entre d'une part le Cameroun et le Nigeria et d'autre part Yaoundé et N'Djamena à propos de la détermination de la paternité et du tracé de l'embouchure du Chari, la convention de démarcation est approuvée en 1994 par les chefs d'Etat de la CBLT. Malheureusement, sa ratification par pays, prévue avant le sommet de N’Djamena des 30-31 octobre 1996, s'est soldée par un échec à cause de l'opposition du Nigeria. Le statu quo se voit maintenu jusqu'à l'Arrêt de la CIJ de 2002 qui confirme les travaux de la CBLT 25.

21. D’une manière générale, le lac Tchad a fait l'objet d'une délimitation précise, par traités, entre Allemands, Français et Anglais. Elle est le résultat de la superposition d'accords successifs conclus entre les puissances coloniales à la fin du $\mathrm{XIX}^{\mathrm{e}}$ siècle et au début du $\mathrm{XX}^{\mathrm{e}}$.

22 . Moisel était l'officier allemand chargé d'établir les cartes administratives du protectorat allemand du Kamerun. Les Français s'appuieront sur ses travaux pour bâtir toute l'organisation territoriale du territoire camerounais qui leur est confiée par le mandat de la SDN après la Première guerre mondiale. Ces cartes serviront de même à la délimitation des frontières externes du Cameroun. Voir Abdouraman H., op. cit.

23. Mondji L., op. cit., pp. 6-7.

24 . "Cameroun-Nigeria : les étapes de la normalisation ", Cameroun Tribune, n7965/4524, $29^{\mathrm{e}}$ année, 3 novembre 2003, p. 7. Dès décembre 2002, Darrak, Naga, Tchika, Ndjamboumba et les villages environnants redeviennent camerounais. Voir Le Messager, ${ }^{\circ} 1425,22$ décembre 2003.

25. CBLT 0037, PV de la 36 section, Maroua, 30 novembre - $1^{\text {er }}$ décembre 1988. Annexe G. Rapport de la sous-commission génie civil et télécommunication relatif à la démarcation des frontières. Il est également utile de lire l'article de Njeuma M.Z., "Contributions diplomatiques et administratives à la paix sur la frontière entre le Cameroun et le Nigeria », Des frontières en Afrique $d u X I I^{e}$ an $X X^{e}$ siècle, Paris, UNESCO/CISH. (Actes du colloque de Bamako - Mali, 15-19 mars 1999, sur le thème : "Histoire et perception des frontières en 
Comme mentionné précédemment, la frontière dans le lac Tchad est matérialisée par des barres de fer. Visibles en période de décrue, c'est-à-dire de février à septembre, ces barres sont immergées durant tout le reste de l'année. Mais il faut bien se rendre compte que la démarcation effective des limites internationales ne gêne nullement les populations, qui se meuvent sans tenir compte de ces barrières. L'application du verdict pourrait donc venir compliquer les choses.

La mise en œuvre de l'Arrêt du 10 octobre 2002, a été confiée à la Commission mixte Cameroun-Nigeria. Mise en place en novembre 2002 à Genève, lors de la rencontre entre les présidents Paul Biya et Olusegun Obasanjo, sur invitation du Secrétaire général de l'Organisation des Nations unies, Kofi Annan, elle permettra la rétrocession des territoires camerounais occupés par le Nigeria 26.

$\mathrm{Si}$, dans l'ensemble, le retrait des forces nigérianes de Darak s'est bien déroulé, il subsiste des problèmes de leadership qui pourront dégénérer en affrontements s'ils sont négativement instrumentalisés. Il s'agit particulièrement de ces élites nigérianes de la presqu'île de Darak qui n'entendent pas perdre les avantages accumulés depuis plus de quinze ans. Les propos de Mal Garba, d'ethnie haoussa du Nigeria et chef d'un quartier à Darak depuis l'occupation nigériane, sont édifiants à cet égard :

«Si le Nigeria accepte la décision de la Cour [...], nous n'aurons d'autre choix que de rejeter cela complètement. Ils devront traverser sur nos cadavres. Le Cameroun ne pourra jamais administrer cette terre, tant que nous sommes en vie [...]. Rien sur la surface de la terre ne pourra nous soumettre au gouvernement camerounais. Et si le Nigeria nous lâche, nous n'aurons pas d'autre choix que nous accrocher à nos terres par tous les moyens. Je dis bien, par tous les moyens ${ }^{27}$.»

Contrairement à ce que laissent entendre les propos de Mal Garba, la communauté de Darak va continuer de vivre dans la quiétude, malgré le transfert d'autorité des chefs nigérians aux Camerounais. D'ailleurs, le cosmopolitisme de la région aboutit davantage à une négation de l'origine ethnique ou étatique. Néanmoins, les autorités camerounaises, comme pour éviter toute nouvelle prétention territoriale du Nigeria sur la zone, décident de passer à une présence effective. En effet, le président Paul Biya érige Darak en chef-lieu d'arrondissement le 29 janvier 2004. Cette mesure entre en droite ligne de la théorie administrative du rapprochement de l'administration des administrés, bien qu'en réalité, ce soit l'Administration qui avait besoin de ces derniers, car elle était complètement absente, d'où l'occupation militaire et administrative

Afrique du XII ${ }^{\mathrm{e}}$ au XXe siècle dans le cadre d'une culture de la paix »), 2005, pp. 159-189.

26. Cameroun Tribune, $\mathrm{n}^{\circ} 7965 / 4524$, op. cit.

27. Mal Garba, interrogé le 4 janvier 2004 à Darak par Mondji Lucas. Voir : www.icj-cij.org 
de la zone par le Nigeria. Ce faible encadrement administratif est observable dans presque toutes les zones frontalières du Cameroun septentrional.

Ainsi, les différends frontaliers avec le Nigeria ont poussé le gouvernement camerounais à être effectivement présent dans les zones frontalières. La création de l'arrondissement de Darak entre donc dans l'optique de la sécurisation des frontières internationales du pays. Son achèvement doit s'accompagner d'infrastructures sociales, susceptibles de promouvoir le développement de la zone. L'installation des postes de police et d'un peloton de gendarmerie est un signe visible de la prise en main de la région par l'Etat camerounais.

Le 6 juin 1994, profitant de l'enlisement du «conflit de Bakassi 28 ", le Cameroun décide alors de clarifier toute sa frontière le séparant de son géant voisin devant la Cour internationale de Justice (CIJ). A l'issue de l'arrêt de la chambre en 2002, Darak est confirmée camerounaise et érigée la même année en chef-lieu d'une nouvelle circonscription administrative. Malgré les différentes rétrocessions de territoires constatées depuis cette date, l'île dont il est question dans cette réflexion est toujours sous domination économique nigérianne ${ }^{29}$. Les populations camerounaises de cette région semblent s'accommoder de cette prépondérance "étrangère ", au regard du contexte de paix dans lequel se trouve Darak depuis la rétrocession, contrairement à la région de Bakassi, théâtre de nombreux incidents, souvent meurtriers.

Au-delà des considérations locales, il semble nécessaire d'intégrer le problème dans l'analyse globale des politiques des frontières pratiquées par le Cameroun et le Nigeria, et ce d'autant plus qu'il y a bien deux manières de voir les frontières conditionnées par l'histoire, les réalités politiques et sociales internes propres à chaque pays.

\section{Deux Etats, deux politiques de frontière distinctes : dynamisme nigérian ou négligence camerounaise?}

A bien y regarder, tous les conflits nés sur la longue frontière camerounonigériane $(1500 \mathrm{~km})$ et ailleurs semblent tenir au caractère expansionniste du Nigeria et des Nigérians d'une part, et à la léthargie de l'Etat camerounais en matière de politique des frontières d'autre part.

En effet, comme l'a encore si bien reconnu le président Paul Biya, en s'adressant le 31 décembre 2007 à ses compatriotes (dans le cadre du tradition-

28. Le «conflit de Bakassi» est une dénomination beaucoup plus journalistique qualifiant le conflit frontalier opposant le Cameroun et le Nigeria dans le Golfe de Guinée. Contrairement à Darak où la résolution du conflit est pacifique, à Bakassi, du fait des potentialités pétrolières de la région, des affrontements armés ont secoué le processus de pacification.

29. Voir dans cet article le tableau détaillant les statistiques des commerçants qui contrôlent le trafic du poisson dans l'arrondissement. 
nel message du nouvel an), les frontières du Cameroun sont très poreuses. Cette porosité explique largement les situations d'insécurité constatées actuellement sur la frontière séparant le Cameroun de la République centrafricaine ${ }^{30}$. La perméabilité des frontières et toutes les conséquences négatives qui y sont rattachées semblent découler de la tiédeur, voire de ce qui s'apparente à l'absence de politique des frontières au Cameroun.

En effet, depuis l'accession à l'indépendance en 1960, les autorités camerounaises ne se sont pas dotés de programme de développement des zones frontalières. Le premier président du pays s'est surtout attelé à la consolidation d'un Etat fort. Le contexte de rébellion créé par les partisans de l'Union des populations du Cameroun (UPC) appelait au développement d'une politique d'unité nationale qui sera le credo de tout le régime d'Ahmadou Ahidjo. Même s'il faut signaler la participation du Cameroun dans l'institution de la CBLT pour pacifier les relations transfrontalières dans le lac Tchad, il n'en demeure pas moins que la question des frontières a été plus ou moins laissée de côté. Il faudra en effet attendre 1985 pour voir le pays se doter d'un organisme spécialisé s'y rattachant.

Depuis sa création, la Commission nationale des frontières (CNF) n'a pas véritablement fonctionné malgré l'importance que revêtent les zones frontalières dans la stabilité interne et le développement économique. Adamou Ndam Njoya, ancien ministre de l'Education nationale et actuel président de l'Union démocratique camerounaise (UDC), parti d'opposition, a résumé l'état d'esprit qui aurait empêché la mise en œuvre d'une politique des frontières féconde en 2002 :

"Quand la Commission des frontières avait été créée, il y a plus de 25 ans, j'avais dit qu'il fallait aller occuper le terrain. A l'époque, on avait peur, on croyait que les Nigérians utiliseraient les routes et les infrastructures créées par nous pour nous envahir facilement. C'est un raisonnement fallacieux. Il faut occuper notre terrain. Il faut des fonds spéciaux pour les frontières ${ }^{31}$. 》

Devant l'enlisement du conflit frontalier de Bakassi, le président Paul Biya décide le 27 juillet 2001 de restructurer la Commission nationale des frontières (CNF). Les missions qui lui sont assignées la rattachent au Secrétariat général de la présidence. Ainsi, on peut lire dans les différents articles du décret qu'elle est :

30. Nous faisons allusion à la migration forcée des éleveurs mbororo menacés par des attaques des « coupeurs de route » (brigands de grands chemins) qui se transforment désormais en preneurs d'otages. Des enquêtes réalisées sur le terrain, il ressort que ces derniers sont issus des rangs des soldats tchadiens ayant aidé le général Bozizé d'origine gbaya (ethnie répartie de part et d'autre de la frontière RCA-Cameroun) à prendre le pouvoir à Bangui.

31. Bambou F., "Bakassi: Comment Paul Biya abandonne les frontières ", La Nouvelle Expression, 18 août 2006. 
« [...] un organisme consultatif chargé de formuler des propositions au gouvernement sur toute question se rapportant à la conception, à la coordination et à la mise en ouvre de la politique nationale des frontières. A ce titre, elle assure en liaison avec les départements ministériels et les services concernés toutes études, toutes actions de suivi et de sensibilisation relatives à la définition, à l'effectivité, à la stabilité et à la valorisation des frontières nationales. Elle est rattachée au Secrétariat général de la présidence de la République ${ }^{32}$.

Par ailleurs, malgré son pouvoir consultatif, l'organe des frontières est doté d'un conseil. Celui-ci doit se réunir au moins quatre fois par an. En outre, elle est composée de nombreux ministres (Administration territoriale, Justice, Garde des sceaux, Relations extérieures, Défense, Cadastre et mines), du secrétaire d'Etat chargé de la Gendarmerie, du secrétaire général des services du Premier ministre, du délégué général de la Sûreté nationale, du directeur général de la Recherche extérieure ainsi que de tout autre ministre dont la présence peut s'avérer utile. Ce panel d'autorités devait, en pratique, être chapeauté par un secrétariat permanent de la CNF. Ses membres sont des cadres de haut niveau.

Cependant, ces dispositions institutionnelles ne sont pas toujours mises en œuvre. Depuis son institution et surtout sa restructuration en 2001, la Commission n'a pas de secrétaire permanent. Qui plus est, le Conseil de supervision ne s'est jamais tenu.

Or, des dispositions transitoires et pratiques ont été initiées depuis l'arrêt de la CIJ pour développer les zones frontières camerounaises et sécuriser ainsi les frontières internationales. Il s'agit notamment de la création de l'arrondissement de Darak en janvier 2004, suivi de celui de Kye-Osi en 2006 sur la frontière séparant le Cameroun de la Guinée équatoriale et du Gabon. De plus, des postes de santé frontière sont théoriquement créés depuis 2006. Le renforcement de la surveillance des frontières se dessine peu à peu à travers la création de plusieurs postes de police. Enfin, l'assassinat de 15 soldats camerounais, par des groupes non identifiés à la fin de l'année 2007, dans la péninsule de Bakassi, a amené le président Paul Biya à doter Darak, Kyo-Si et Bakassi de brigades de gendarmerie ${ }^{33}$. Cette sécurisation de la frontière qui se développe par à-coups ne permet pas de dire que le Cameroun a une réelle politique des frontières. Or, c'est le contraire de ce qui se passe au Nigeria ${ }^{34}$.

32. Ibid.

33. Journal radiodiffusé par la CRTV, média officiel au Cameroun.

34. Ce tâtonnement se clarifie davantage au fur et à mesure que l'on approche du 14 août 2008, date à laquelle s'officialise la rétrocession totale de l'île de Bakassi (occupée par le Nigeria) à l'autorité camerounaise. Pour saluer cette résolution pacifique - rare en Afrique noire - les organismes internationaux décident d'appuyer le Cameroun dans la viabilisation de ces zones frontalières. Ainsi, la Belgique assistera Yaoundé sur deux ans (2008-2010) dans l'électrification de 26 villages situés le long de la frontière avec le Nigeria. 
Signalons avant tout que le géant d'Afrique, comme son voisin le Cameroun et bien d'autres pays d'Afrique subsaharienne, a connu deux phases dans la définition de sa politique des frontières : de l'indépendance à 1987, et de 1987 à nos jours. La première période correspond au souci d'affirmation de la souveraineté nationale. Dès lors, comme le remarque Anthony Asiwaju, les zones frontalières sont alors considérées comme "une périphérie nationale, politiquement désavantagée du fait de sa vulnérabilité militaire 35 ». Par conséquent, ces no man's lands dont tout le monde se méfie sont longtemps restés à l'écart des investissements, ce qui explique leur sous-développement général.

Naturellement, le nationalisme qui tend vers un protectionnisme ne peut que crisper les relations transfrontalières. C'est pourquoi dans presque toute l'Afrique subsaharienne, cette période est marquée par de nombreux conflits frontaliers ${ }^{36}$. Il en va ainsi de ceux nés sur la frontière maritime entre le Nigeria et le Cameroun et qui ont failli dégénérer en une guerre ouverte entre les deux pays en 1982 et, bien sûr, le conflit armé entre le Nigeria et le Tchad, d'avril à juin 1983. Arguant de « la guerre contre l'indiscipline » (contrebande, trafic des devises, activités illégales, etc.), les autorités nigérianes décident, d'avril 1984 à mars 1986, de la fermeture et de la sécurisation militaire de toutes les frontières du Nigeria ${ }^{37}$. Cette réaction par salves, à l'image de ce que font actuellement les dirigeants camerounais, témoigne d'une absence de politique des frontières.

Un mouvement de protestation politico-sociale interne force les autorités nigérianes à rouvrir les frontières. Aussi, sur la base des études multidisciplinaires faites par le National Institute for Policy and Strategic Studies (NIPSS) de Kuru et relatives à la recherche d'une politique de sécurité et de défense des frontières internes et externes, le Nigeria met sur pied la National Boundary Commission (NBC). Contrairement à la CNF camerounaise créée deux ans plus tôt et qui n'a jamais fonctionné ${ }^{38}$, la NBC nigériane est au-delà d'un pur organe de consultation. En effet, Anthony Asiwaju souligne que la NBC :

«[...] est un organisme exécutif fédéral s'appuyant sur la Constitution et doté de grands pouvoirs. Sa mission est "de se saisir de tout litige frontalier pouvant surgir entre le Nigeria et n'importe lequel de ses voisins, on entre deux Etats de la Fédération, de trancher et d'intervenir en vue de régler le différend". Créée par le décret 38

35. Asiwaju A.-I., «Fonctionnement et gestion des pays frontières en Afrique de l'Ouest: L'expérience vécue du Nigeria et de ses voisins immédiats », Communication faite à la réunion spéciale du Club du Sahel et de l'Afrique de l'Ouest sur le thème "Pour une meilleure approche régionale du développement ", deuxième séance : «Les pays frontières », Accra (Ghana), 20-21 mai 2002.

36. La médiatisation des conflits frontaliers interethniques des années 1990, notamment ceux de la zone des Grands Lacs, ont rapidement mis entre parenthèses les litiges de frontières des années 1980. Or, ils sont à la base des différends frontaliers constatés à la fin du XXe siècle.

37. Asiwaju A.-I., "Fonctionnement et gestion des pays frontière en Afrique de l'Ouest: L'expérience vécue du Nigeria et de ses voisins immédiats », op. cit. 
du 17 décembre 1987 et officiellement entrée en fonction le 20 juillet 1988, la Commission est également appelée à mettre en place des mécanismes et programmes susceptibles de prévenir les conflits frontaliers et de promouvoir la coopération transfrontière. La coopération transfrontière est considérée comme une priorité dans le décret d'application qui, entre autres, habilite la Commission à "créer des comités chargés de se saisir des questions touchant aux frontières du Nigeria avec les pays limitrophes, qu'il s'agisse de questions politiques, économiques ou juridiques ou de questions ayant trait au développement et à la sécurité des régions frontalières" 39 ».

Ainsi, la NBC composée de onze membres réguliers et placée sous la supervision du vice-président est dotée d'un secrétariat permanent logé à la présidence. Ce dernier assure la coordination. Néanmoins et contrairement à la CNF, il a été :

«[...] doté de pouvoirs très étendus et bénéficie de financements conséquents alloués par le gouvernement. Bien qu'il ne s'agisse pas, de par la loi, d'un secrétariat exécutif, il est placé sous l'autorité d'un directeur général, fonctionnaire ayant rang de secrétaire permanent dans la fonction publique fédérale. Le secrétariat est constitué de quatre grands départements : Frontières internationales, Frontières inter-Etats (interne), Centres de recherche et de documentation, Personnels et finances ${ }^{40}$. »

Muni de cet instrument de gestion des frontières, le Nigeria est alors à l'avant-garde de la question de l'intégration régionale. Il s'est fixé trois priorités, à savoir la "coopération transfrontière vigoureuse et systématique avec instauration de la confiance; développement des pays frontière ; et tracé des frontières 41 ». Il s'agit pour la première de l'organisation d'une série d'ateliers consultatifs de coopération bilatérale transfrontière puis des missions d'instauration de confiance avec les pays voisins, dont le Cameroun. Ainsi, des tournées ont été réalisées dans les zones frontalières par le ministre nigérian des Affaires intérieures et ses homologues. Il est même à signaler la création d'un ministère de la Coopération et de l'Intégration en Afrique ${ }^{42}$.

Quant au développement des " pays frontières » (régions frontalières) initié en 1989, il s'est concrétisé par la création du National Border Region

38. Depuis sa création, la CNF n'a jamais tenu de session. D’ailleurs, la nomination de son secrétaire exécutif est toujours attendue.

39. Bambou F., « Bakassi : Comment Paul Biya abandonne les frontières », op. cit.

40. Ibid.

41. Asiwaju A.-I., «Fonctionnement et gestion des pays frontière en Afrique de l'Ouest: L'expérience vécue du Nigeria et de ses voisins immédiats », op. cit.

42. Ibid. 
Development Committee (NBRDC). Elle a abouti à la mise sur pied d'un fonds pour le développement des communautés frontalières qui s'élevait en 2002 à hauteur prévisionnelle de 25 millions de dollars US.

La dernière priorité de la NBC porte sur les tracés des frontières. Concernant les résultats de cette orientation, Anthony Asiwaju signale que :

«[...] l'approbation, non sans les demandes d'informations d'usage déposées par les Etats membres (notamment le Nigeria, le Tchad et le Cameroun), des travaux de tracé effectués dans le bassin du lac Tchad sous l'autorité de la Commission de ce même bassin ; la reprise en mars 1989 des travaux de tracé interrompus depuis longtemps sur la frontière terrestre entre le Nigéria et le Bénin avec des résultats extrêmement positifs ; la reprise des travaux de jalonnement sur la frontière entre le Nigeria et le Niger; les remarquables accords conclus sur la délimitation réelle de la frontière maritime entre le Nigeria et la Guinée équatoriale et enfin, la reprise des négociations sur la frontière maritime entre le Nigéria et le Bénin ${ }^{43}$ ».

C'est donc pourvu de cette structure de réflexion et d'action que le Nigéria développe un programme de développement des régions frontalières qui s'apparente à une politique expansionniste. C'est pourquoi, de la fin des années 1970 au début du XXIe siècle, l'administration nigériane n’hésite pas à occuper naturellement la région camerounaise de Darak.

Ainsi, il apparaît clairement que le conflit frontalier qui a opposé le Cameroun au Nigeria tient compte de plusieurs facteurs liés aux politiques et pratiques frontalières des deux Etats, de même qu'aux enjeux géopolitiques et géo-économiques relatifs au retrait des eaux du lac Tchad qui appellent à une gestion négociée du principe de l'intangibilité des frontières héritées de la colonisation européenne. D'ailleurs, avant, pendant et après l'occupation nigériane de Darak, la population a toujours développé des relations permanentes le long des rives du lac Tchad, en dépit des considérations étatiques.

\section{Continuité du système de coopération économique et sociale entre les populations de Darak pendant et après l'occupation nigériane : le lac Tchad, un espace international}

Le bassin tchadien est donc un espace qui s'accommode fort peu des frontières étanches. L'attrait de l'eau et des ressources qui y sont liées font que les migrations humaines conditionnent toutes les relations sociales. La diminution progressive de la surface des eaux du lac a poussé les populations à se défaire des frontières. C'est le 
cas des Nigérians, Maliens, Tchadiens, Centrafricains et autres, qui ont choisi de s'installer dans le nouvel eldorado des éleveurs, agriculteurs et, surtout, pêcheurs qu'est Darak.

L'occupation nigériane de Darak obéit à cette logique historique dictée par une nécessité sociale et économique ${ }^{44}$. Et cette réalité historique se traduit clairement par la continuité et l'intensification des échanges entre le Cameroun et le Nigéria dans cette région depuis décembre 2003, date du début de retrait du Nigéria du territoire disputé ${ }^{45}$.

En effet, le commerce du poisson vers le Nigéria est le baromètre qui permet ici de saisir la dynamique de coopération qui existe entre les habitants de cette région. Signalons avant tout que c'est le commerce qui va favoriser l'immigration massive des Nigérians vers Darak. La composition démographique l'exprime parfaitement. D'une population ayant rarement atteint le millième, le chef-lieu de l'arrondissement de Darak comptait en 2005 environ 20000 habitants. Le tableau suivant nous donne un aperçu de l'importance démographique de certaines îles de l'arrondissement de Darak.

Tableau 1 : Situation démographique de l'arrondissement de Darak en 200546

\begin{tabular}{|l|l|}
\hline Îles & Nombre d'habitants \\
\hline Darak 1 & 20000 \\
\hline Nimeri & 6000 \\
\hline Karakaya & 2500 \\
\hline Katikime 2 & 1000 \\
\hline Darak 2 & 250 \\
\hline Gore Tchandi & 2500 \\
\hline Bouaram & 1000 \\
\hline Total & 31081 \\
\hline
\end{tabular}

La densité de population est assez importante pour ces îles, le plus souvent de petite taille et flottantes ${ }^{47}$. D'ailleurs, la plupart des arrondissements camerounais du département du Logone-et-Chari (Kousseri) dépassent rare-

44. Rappelons tout de même que ce mouvement migratoire vers le Cameroun était plutôt orienté vers le Nigeria avant les sécheresses des années 1970-1980. C'est du fait du retrait des eaux que les ressources halieutiques nigérianes se sont éteintes, poussant les poissons à migrer vers la région de Darak. 45. Abdouraman T., op. cit.

46. Source : Archives de la sous-préfecture de Darak, citées par Abdouraman T., op. cit., p. 26.

47. Ces îles sont en effet flottantes compte tenu du fait qu'elles sont fixées sur du sol mouvant. 
ment les 13 habitants au $\mathrm{km}^{2}$, tandis que Darak 1, à elle seule, détient au moins 83 habitants au $\mathrm{km}^{2} 48$.

La part occupée par les Nigérians dans la démographie est très intéressante. En effet, selon des sources officielles - venant de la sous-préfecture de Darak - les Camerounais représentent moins de $10 \%$, tandis que les Nigérians représentés dans cette zone par les Haoussa (49\%), les Margui (16\%), les Djoukoun (15\%) et les Bornouan ou Kanuri (8\%) en constituent la majorité ${ }^{49}$. Ainsi, les chiffres indiquent clairement une suprématie des Nigérians, malgré l'arrêt de la CIJ pour la paternité du Cameroun sur Darak. Cette domination numérique se traduit également par un contrôle presque total du circuit commercial autour du poisson.

La pêche constitue le fondement de l'activité économique dans le lac Tchad, bien qu'il faille signaler l'importance de l'élevage et de l'agriculture. D'environ 100000 tonnes de poisson frais capturé au début des années 1970, la production piscicole dépasse 1400000 tonnes depuis l'occupation de Darak par les Nigérians ${ }^{50}$. Cette grande production est essentiellement contrôlée et largement orientée vers Maiduguri, la capitale nigériane du Borno State ${ }^{51}$. Le commerce du poisson nécessite de gros moyens financiers que n'hésitent pas à placer les bailleurs de fonds nigérians compte tenu des bénéfices à en tirer. Le tableau suivant nous aide à mesurer l'impact financier de la commercialisation du poisson fumé, du poisson frais et du poisson séché.

Tableau 2 : Darak, estimation des prix du poisson en juin 2006

\begin{tabular}{|l|c|c|c|}
\hline Unité de mesure & Poisson frais & Poisson séché & Poisson fumé \\
\hline Bassine $(20 \mathrm{~kg})$ & $10000 \mathrm{Fr}$ CFA & $2000 \mathrm{Fr}$ CFA & $3000 \mathrm{Fr}$ CFA \\
\hline Carton & - & - & $200000 \mathrm{Fr}$ CFA \\
\hline Sac $(10 \mathrm{~kg})$ & - & $80000 \mathrm{Fr}$ CFA & $250000 \mathrm{Fr}$ CFA \\
\hline
\end{tabular}

D’ailleurs, de nombreuses terres émergées naviguent au gré des courants. D’où, une fois de plus, le problème de la démarcation des frontières dans le lac Tchad.

48. Abdouraman T., op. cit., p. 26.

49. Ibid., p. 28. Les autres habitants sont originaires de pays lointains.

50. Durang J.-C., Situation actuelle des pêcheries dans la région du lac Tchad, Documents de l'ORSTOM, 1977, cité par Abdouraman T., op. cit., p. 31.

51. Il faut noter tout de même que, contrairement aux destinations camerounaise et tchadienne du poisson dit «Salanga », le Banda est entièrement drainé vers Maiduguri. 
Nous voyons donc l'importance financière liée au commerce du poisson en général et du poisson fumé en particulier, très consommé semble-t-il dans le Sud du Nigéria ${ }^{52}$. Ainsi, le poisson fumé, le poisson frais et le poisson séché à Darak au Cameroun est vendu à hauteur de $80 \%$ à Maiduguri. En moyenne, des dizaines de camions de 9 tonnes alimentent ce marché tous les mercredis et jeudis. On estime la valeur numéraire de ce commerce à plus de 7000000 F CFA par semaine. Et dans ce système, les commerçants nigérians sont majoritaires, comme le montre ce tableau.

Tableau 3 : Nationalités et spécialités des commerçants du poisson de Darak en fin $2006{ }^{53}$

\begin{tabular}{|l|l|l|l|l|l|}
\hline $\begin{array}{l}\text { Nationalités } \rightarrow \\
\text { Specialités } \downarrow\end{array}$ & nigériane & camerounaise & tchadienne & malienne & autres \\
\hline grossistes & 2442 & 1053 & 1000 & 900 & 942 \\
\hline Intermédiaires & 1500 & 250 & 250 & 500 & 742 \\
\hline Détaillants & 1542 & 342 & 342 & 300 & 328 \\
\hline
\end{tabular}

Nous observons une certaine continuité de la coopération économique née grâce à l'occupation nigériane. Cette dynamique est progressive et, en somme, bénéfique aux deux pays, dans la mesure où, depuis la création de l'arrondissement de Darak en 2004, le Cameroun en tire des devises relatives aux différentes taxes. Les autorités nigérianes, de leur côté, gagnent à pérenniser ce commerce qui est détenu à plus de $80 \%$ par leurs ressortissants. C'est peut-être la conjugaison de ces intérêts mutuels interconnectés qui justifie le règlement pacifique de ce conflit, ce qui souligne encore le caractère international du lac Tchad 54 .

\section{Pour des frontières internationales pacifiées et pacifiantes, les leçons d'une intégration par le bas}

Au terme de cette réflexion, il s'en dégage une lueur d'espoir, bien que, de part et d'autre de la frontière camerouno-nigeriane, se développent des politi-

52. Abdouraman T., op. cit., p. 50.

53. Ibid.p. 54.

54. Dans un article intéressant, Agnès Lambert décrit la contribution des commerçants dioula dans la facilitation de l'intégration ouest-africaine. Voir Lambert A., « Les commerçants et l'intégration régionale ", in Diop M.-C., Le Sénégal et ses voisins, Paris, SET, 1994, pp. 81-94. 
ques internes visant à la rendre plus étanche politiquement. Mais les deux Etats n'ont d'autre choix que de coopérer, les ressources du lac Tchad se raréfiant de jour en jour. Les exemples du poisson, des espaces agricoles, des pâturages et même de l'eau sont significatifs.

Cependant, le retrait (irréversible) des eaux du lac Tchad, si les mesures en cours à la CBLT ne sont pas appliquées - notamment le transfert des eaux de l'Oubangui vers le lac - est un enjeu capital dans les relations entre les deux Etats. Somme toute, en dépit de la volonté manifeste pour une intégration africaine, le besoin de sécurité transfrontalière et donc de relations transfrontalières pacifiques passerait avant tout par une acceptation des frontières héritées de la colonisation. C'est, nous semble-t-il, la condition sine qua non d'une paix aux frontières interétatiques. Toute remise en cause de cet héritage colonial aura un effet domino. D'ailleurs, les prétentions expansionnistes nigérianes tiennent beaucoup à cette négation du passé colonial.

Même s'il faut démarquer clairement l'espace de chaque nation dans le lac Tchad, il importe que les autorités étatiques s'accordent à la définition des mesures de coopération visant à une gestion partagée des ressources du lac 55 .

Mais les populations frontalières ont depuis longtemps appliqué la politique de coopération transfrontalière. Le cas de l'exploitation des ressources halieutiques de l'arrondissement de Darak par les négociants nigérians au moment où les deux pays se diputent sa paternité témoigne d'une intégration par le bas. Celle-ci tient à des facteurs économiques, historiques et socioculturels qui ont toujours fait fi des frontières. Il est donc important que les dirigeants des deux pays, tout en s'assurant de leur souveraineté mutuelle, mettent au point des mécanismes de pacification de leur frontière. Cette dernière, malgré la mise en œuvre concertée de l'arrêt de la CIJ de 2002, est toujours « conflictogène ».

55. L'appel du professeur Anthony Asiwaju relatif à la vulgarisation du développement des «pays frontières » est à promouvoir (voir Asiwaju A.-I., op. cit.). 